\title{
Essai de synthèse des connaissances en érosion et hydraulique torrentielle
}

\author{
M. Meunier
}

CEMAGREF Grenoble

\section{Introduction}

Sous le thème érosion et hydraulique torrentielle, sont regroupées quelques disciplines scientifiques qui vérifient simultanément les critères suivants :

- être quantitatives ;

- avoir pour objet d'étude la formation et la propagation de débits liquides et de débits solides, avec leurs interrelations ;

- avoir pour terrains d'application les bassins versants de montagne et les torrents (donc non compris les rivières torrentielles).

Dans la pratique courante, par extension des modes de raisonnement utilisés pour les bassins versants et les cours d'eau de plaine, on sépare souvent ce qui traite des débits liquides et ce qui traite du transport solide ; en fait, les interrelations, notamment dans la propagation, sont souvent fortes et cette séparation n'est plus valable pour les phénomènes paroxysmaux, alors qu'on peut malgré tout la conserver, au moins sur un plan conceptuel, pour la formation des débits; on peut donc présenter toutes les disciplines qui correspondent aux trois critères ci-dessus sous la forme du tableau suivant :

\begin{tabular}{|c|c|c|c|}
\hline $\begin{array}{r}\text { Caractéristi- } \\
\text { ques et } \\
\text { lieu d'ap } \\
\text { plica- } \\
\text { tion }\end{array}$ & $\begin{array}{c}\text { Versants } \\
\text { torrentiels. } \\
\text { Formation } \\
\text { des débits } \\
\text { débit }\end{array}$ & $\begin{array}{c}\text { Evolution des } \\
\text { débits dans } \\
\text { le réseau } \\
\text { hydrographique }\end{array}$ & $\begin{array}{c}\text { Ensemble du } \\
\text { bassin versant } \\
\text { considéré comme } \\
\text { un système } \\
\text { et pris } \\
\text { dans sa totalité }\end{array}$ \\
\cline { 1 - 2 } Débit liquide & $\begin{array}{c}\text { HYDROLOGIE } \\
\text { TORRENTIELLE }\end{array}$ & HYDRAULIQUE & DYNAMIQUE \\
\cline { 1 - 2 } Débit solide & EROSION & TORRENTIELLE & TORRENTIELLE TORRENTIELLE \\
\hline
\end{tabular}

Notre thème exclut en fait l'hydrologie torrentielle parce que c'est une discipline qui peut être individualisée ; on peut en effet, au moins dans les cas courants, représenter la formation des débits sur les versants par le schéma suivant où les flèches désignent des relations de cause à effet :

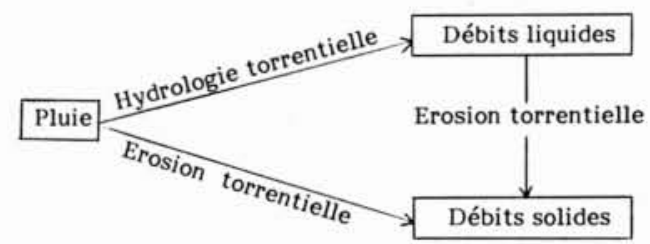

\section{Attempt of synthesis of the knowledge in mountain erosion and torrential hydraulics}

The introduction makes explicit what should study erosion and hydraulics sciences in mountain catchments; then, characteristics and methods of torrential erosion are described, as well as research projects which are going on in France. In a second part, are explained the differences between torrential and classical hydraulics; then, two theories used for debris flows, and at last a set of formulas which allow the engineer to compute solid and liquid discharge as well as the stage of the mixture in erodible torrents. 
Ce schéma montre que si en érosion torrentielle on a besoin des résultats de l'étude hydrologique, l'inverse n'est pas vrai et l'hydrologie torrentielle peut être traitée indépendamment des érosions qu'elle génère. De plus, bien que peu pourvue en données en comparaison de l'hydrologie de plaine, l'hydrologie de montagne en possède malgré tout infiniment plus que le spécialiste en érosion; ces deux disciplines n'en sont donc pas au même degré d'avancement ; leur séparation est d'ailleurs une bonne chose car cela a permis à l'hydrologie de progresser sans être hypothéquée par le manque de données relatif à l'érosion de montagne.

La séparation entre débits liquides et débits solides, envisageable sur les versants, ne peut plus être acceptée dans le réseau hydrographique dès que le transport solide est important ; c'est-à-dire, pour l'aménageur, dès que les questions pertinentes deviennent relatives à la quantité de sédiments, à leur éventuel dépôt, à l'obstruction des ponts, etc... ou pour l'hydraulicien un peu plus théoricien, dès que le volume de sédiments influe sur la hauteur d'eau ou sur la vitesse du fluide, rendant par cela même inopérantes les formules du type Manning-Strickler ou Chézy. Dès lors, l'hydraulique torrentielle gagne un degré important de complexité par rapport à l'hydraulique usuelle pratiquée par les ingénieurs de terrain puisqu'on se trouve d'emblée dans une hydraulique biphasique sans qu'on puisse considérer une phase comme marginale par rapport à l'autre.

Après l'érosion torrentielle, après l'hydraulique torrentielle, le troisième volet de notre tableau est la dynamique torrentielle où l'imbrication entre le débit liquide et le débit solide est une évidence, car dans les torrents, les discontinuités de fonctionnement qui interviennent brutalement entre deux périodes de relative stabilité, affectent surtout la présence du sédiment, que ce soit sous l'effet d'un phénomène naturel (purge brutale du sédiment par une lave torrentielle) ou de l'art de l'ingénieur (rétention du transport solide par construction d'un barrage). Les travaux de recherche existant dans ce volet ne seront pas explicités dans cet essai de synthèse.

\section{Erosion torrentielle}

\subsection{Champ d'étude}

Par érosion torrentielle, nous entendons ici l'étude des phénomènes érosifs de surface d'origine hydrique se produisant sur un bassin versant torrentiel.

Signalons toutefois que l'ingénieur de terrain, confronté à des problèmes de risques naturels sur un bassin versant torrentiel, a adopté une signification plus large due à l'interrelation qui existe entre l'érosion torrentielle au sens strict et les autres processus érosifs : un affouillement de berge peut activer un glissement de terrain, les avalanches amènent des sédiments dans le réseau hydrographique qui seront repris par les crues. Une étude d'aménagement d'un bassin versant torrentiel doit donc s'intéresser à l'ensemble des processus érosifs, qui est souvent alors désigné sous le terme d'érosion torrentielle.

\subsection{Caractérisation}

En tant que discipline scientifique, l'érosion torrentielle se caractérise par les faits suivants :

Absence d'appareil de mesure simple et généralisable : les faits expérimentaux naturels sont donc connus qualitativement seulement, et souvent, à travers des témoins occasionnels dont les relations sont parfois douteuses.

Les phénomènes à étudier se produisent dans le milieu naturel : ils ont donc une très grande variabilité naturelle, ce qui pose deux questions méthodologiques difficiles à résoudre :

a) Dans quelle mesure peut-on transposer des résultats d'observations d'un site à un autre ?

b) Dans quelle mesure peut-on reconstituer le milieu naturel au laboratoire pour pouvoir l'étudier efficacement (problème posé pour l'utilisation des gigantesques simulateurs de pluie qui se trouvent dans certains laboratoires, par exemple)?

Les phénomènes à étudier dépendent d'un ensemble de paramètres qui ressortent d'autres disciplines scientifiques telles que la climatologie, l'hydrologie, la géologie, la mécanique des sols, l'altérologie, la botanique ; on en déduit ceci :

a) Pour faire progresser l'érosion torrentielle par la méthode statistique, il faut un grand nombre de mesures de l'érosion (or, il n'y a pas d'appareil), mais en plus, il faut mesurer un grand nombre d'autres paramètres, chacun d'eux étant susceptible de devenir le paramètre explicatif.

b) Pour faire progresser l'érosion torrentielle par la méthode expérimentale, il faut pouvoir isoler des sites où seul un paramètre explicatif sera responsable de l'érosion, les autres étant fixés à une valeur connue ; d'où bien sûr l'idée d'opérer en laboratoire où tous les paramètres peuvent être maitrisés ; mais également la difficulté d'étudier l'érosion par cette méthode qui conduit à multiplier les cas à étudier.

Les phénomènes à étudier sont vraisemblablement des phénomènes à seuil; ces seuils délimitent des domaines qui devraient être précisés au préalable (classe de pente, classe de couverture végétale, type de sol, etc...) chaque fois qu'on parle d'érosion pour être sûr qu'on parle de la même classe de phénomènes.

L'échelle spatiale des phénomènes est hétérogène; et suivant le cas, on pourra admettre que la quantité érodée est grosso modo proportionnelle à la surface, alors que dans d'autres c'est un détail à l'échelle spatiale qui conditionne le résultat de l'érosion.

On déduit de cette constatation que l'examen du terrain est indispensable et qu'à ce titre le rôle de «l'expert » est irremplaçable.

\subsection{Les méthodes d'étude}

On distingue plusieurs approches.

2.3.1. Approche naturaliste: C'est la démarche qui consiste à étudier le milieu naturel dans sa complexité et à la caractériser au mieux ; elle est pratiquée naturellement par les géologues confrontés à des problèmes 
d'érosion et implicitement par les ingénieurs de terrain confrontés à des problèmes de risque naturel.

Sous sa forme la plus achevée, elle se présente en plusieurs étapes :

a) Cartographier l'espace selon les paramètres pertinents de l'érosion : classe de pente, type de sol, taux de couverture végétale, dynamisme de l'agriculture, etc...

b) Etablir une classification de l'érodabilité du terrain en croisant les valeurs des paramètres : c'est la partie la plus délicate et la moins scientifique de la démarche; elle fait partie de la responsabilité de l'expert, et à ce titre, elle est peu contestée.

Ce croisement des classes de paramètres définit implicitement les domaines de variation des paramètres dont on soulignait l'importance ci-dessus.

c) Cartographier l'espace selon les résultats de cette grille de classification.

Le résultat de cette approche est donc une carte d'érodabilité (CEMAGREF, 1984); cette démarche n'est pas neuve mais l'outil informatique lui a offert un outil performant sous la forme de la cartographie automatique. Des tentatives existent pour utiliser cette démarche non seulement comme outil de description du milieu naturel, mais comme outil de prévision des formes d'érosion, l'hypothèse étant que, à l'intérieur d'un domaine de variation des paramètres de l'érosion, se produiront les mêmes formes d'érosion (M. Guigo, 1978).

Il n'en reste pas moins que cette démarche n'aboutit pas à un résultat quantitatif utilisable par l'ingénieur de terrain et qu'à ce titre elle doit donc être complétée.

\subsubsection{Approche expérimentale: C'est celle qui consiste} à choisir un domaine assez bien délimité de variation des paramètres de l'érosion, à trouver un site naturel lui correspondant ou à reconstituer un tel site au laboratoire et à mesurer les quantités érodées qui pourront ainsi être expliquées en fonction de la variation des autres paramètres.

C'est ainsi que de nombreux laboratoires hors de France utilisent des simulateurs de pluie, parfois très grands, qui leur permettent de connaître l'érosion hydrique sur tel type de sol, à telle pente, etc... On trouve les résultats de telles études dans les comptes rendus de Symposium spécialisés, mais il ne semble pas qu'elles aient permis jusqu'ici l'élaboration d'une théorie, ou même d'une méthodologie générale à laquelle se référer pour la confronter à de nouvelles mesures. L'utilisation de cette approche semble avoir donné par contre des résultats plus intéressants en érosion agricole.

L'autre voie est celle des bassins versants représentatifs expérimentaux où, sur un site particulier, on mesure à la fois l'érosion et les paramètres hydroclimatiques. Leur gros intérêt est que les résultats de mesure concernent un site naturel, et qu'à ce titre, ils sont bien plus crédibles que des résultats de mesure sur site reconstitué au laboratoire ; ils ont par contre des inconvénients dont le plus important est d'être très lourds en investissement et en fonctionnement, ce qui empêche leur multiplication pourtant nécessaire. De plus, se pose le problème de leur représentativité lié à la variabilité minéralogique et géologique qui n'est pas négligeable.
On peut peut-être classer dans cette rubrique la méthode qui consisterait à rassembler toutes les mesures ponctuelles faites à droite et à gauche, et à tenter d'en tirer une théorie synthétique ; cette méthode ne semble pas avoir été mise en œuvre, et elle semble a priori vouée à l'échec vu l'hétérogénéité des mesures de ce type due à celles des appareils de mesure, des organismes producteurs de données, des échelles de temps et d'espace, etc...

2.3.3. Approche empirique : Les démarches précédentes qui peuvent être qualifiées de scientifiques, n'ont pas encore donné un outil à l'ingénieur de terrain pour dimensionner ses ouvrages ; celui-ci reste donc confronté au problème crucial de la prédétermination de la quantité érodée et il se sert, en règle générale, de ce qu'il est convenu d'appeler la méthode historique qui consiste à conserver la mémoire des épisodes importants sur chaque bassin versant torrentiel et à se baser sur eux et notamment le plus catastrophique pour prévoir et dimensionner les ouvrages.

On voit que cette méthode prend directement pour unité spatiale de base le torrent, ce qui permet de sauter toutes les étapes de la démarche naturaliste. Lorsque les enquêtes relatives aux épisodes importants permettent d'aboutir à des volumes globaux de transport solide, il est possible ensuite de les utiliser et de les traduire en formules ou abaques, comme cela s'est fait en Suisse et en Autriche, par exemple.

Un autre type de démarche, qui peut être qualifié d'empirique, est la prolongation de la méthode naturaliste : la carte d'érodabilité est traduite par des ordres de grandeur d'ablation prévisible, ce qui permet à l'ingénieur de terrain de prédéterminer la quantité érodée. C'est ce qui est fait, parait-il, par exemple en Yougoslavie et au Japon.

\subsection{Le point des connaissances en France}

En France, l'art de l'ingénieur s'est exercé depuis très longtemps dans les bassins versants torrentiels et a constitué progressivement une doctrine cohérente, largement empirique, pour dimensionner et concevoir les aménagements de protection. Et si des besoins de connaissance plus quantitative ont toujours été ressentis, ce n'est qu'assez récemment qu'ils ont débouché sur des projets de recherche.

Ces projets se sont portés, pour l'instant, essentiellement sur l'amélioration de notre connaissance de l'érosion des marnes; ils sont traduits sur le terrain par des Bassins Versants Représentatifs sur lesquels sont mesurés outre l'érosion, la pluie, les débits liquides, et les paramètres climatiques; s'il y a un site de Bassins Expérimentaux en terrains glaciaires et schisteux (torrent de l'Eglise, CNRS, LAMA), seuls deux d'entre eux, situés sur des Terres Noires, ont des données d'assez longue durée pour permettre un début de mise en relation des divers paramètres mesurés: il s'agit des BVRE de Draix gérés par le CEMAGREF et l'ONFRTM et des BVRE dits PACA gérés par l'ORSTOM et le BRGM. Les premières démarches ont concerné la nécessité de trouver un appareil pour quantifier soit 
l'altérabilité des marnes (COLAS et DumOlard, 1988), soit les transports solides (MEUNIER et al., 1987, $2^{\mathrm{c}}$ partie). Ceci témoigne bien de la difficulté de cette discipline puisque la méthode de mesures est encore problématique. Par exemple, sur les BVRE de Draix (EGEls et al., 1988), l'écart peut aller de 1 à 3 suivant le type de mesures effectuées.

Par contre, lorsque le procédé de mesures est le même, les résultats sont assez concordants, tant pour l'ablation moyenne annuelle, de l'ordre de 120 à $150 \mathrm{t} / \mathrm{ha} / \mathrm{an}$ (MURA et al., 1988) que pour l'explication du phénomène physique : l'énergie cinétique de la pluie, de préférence après application d'un seuil (BUFALO et al., 1988, Mathys et MEunier, 1988), rend bien compte de la quantité érodée au niveau de l'épisode pluvieux (coefficient de détermination $=R$ de l'ordre de 0,9 ). Sur ces sols marneux, l'effet du reboisement commence aussi à être quantifié (MEUNIER, 1988) : le seuil du ruissellement passe de $10-12 \mathrm{~mm}$ à $25 \mathrm{~mm}$, les volumes sont réduits dans un rapport de l'ordre de 3 à 5 et les pointes de crue dans un rapport de 5 à 10 .

Comme on le voit, ces projets de recherche commencent à apporter des informations intéressantes, mais il leur reste beaucoup à étudier, comme par exemple le phénomène de dépôt et de reprise dans les réseaux hydrographiques qui permettrait d'apprécier le transport solide sur des bassins versants de taille supérieure à la ravine. Mais surtout, il faudrait que d'autres équipes de recherches s'engagent sur d'autres sites de montagne pour qu'on puisse à terme s'appuyer sur des données crédibles et permettre à l'érosion torrentielle de devenir réellement une discipline scientifique quantitative.

\section{Hydraulique et dynamique torrentielles}

\subsection{Introduction}

Par définition, un torrent a une pente forte: à titre indicatif, la classification adoptée dans BERNARD (1927) situe à $6 \%$ la séparation entre une rivière torrentielle et un torrent; en conséquence la quantité de transport solide devient importante et dès $10 \%$, on ne peut plus négliger son influence directe sur la hauteur de l'écoulement ; de plus, à partir d'un certain seuil, le mélange eau + sédiment n'a plus un comportement newtonien. Pour toutes ces raisons, on ne peut plus utiliser telles quelles les formules usuelles de l'hydraulique fluviale.

L'ingénieur de terrain a toujours été conscient de ces difficultés, mais n'ayant pas d'autre outil sous la main, lorsqu'il doit faire des calculs, il utilise les formules de Manning Strickler et de Meyer Peter Muller, en prenant une marge de sécurité bien évidemment. Il ressent particulièrement combien il est démuni face à ces paroxysmes que sont les laves torrentielles, à cause de leur comportement par bouffées, parfaitement inadapté aux calculs classiques qui supposent un régime permanent, et de la hauteur des fronts de lave tout à fait incohérente par rapport aux hauteurs des écoulements constatées avec un débit liquide «normal».

Il se trouve qu'en France, peu de gens se sont penchés sur ces problèmes, du moins dans l'optique de la mécanique des fluides ; mais la recherche à l'étranger est très active et nous allons donc en présenter quelques résultats importants. Nous allons d'abord insister sur le volet « laves torrentielles » qui est encore du domaine de la recherche avant d'expliciter le domaine plus familier des écoulements très chargés pour lequel des outils valables pour l'ingénieur de terrain commencent à se dégager, même si c'est avec une grosse incertitude.

\subsection{Les laves torrentielles}

Comme les laves torrentielles sont souvent issues de phénomènes de nature géologique (glissements de terrain, écroulements), les géologues et géomorphologues s'y sont les premiers intéressés mais, à part quelques exceptions, en se cantonnant à leurs visions des choses, c'est-à-dire en décrivant minutieusement les caractéristiques des déclenchements, des écoulements, des arrêts et des dépôts. Une excellente synthèse bibliographique en langue française a été faite de ce point de vue (SAURET, 1986). Il en ressort que les modalités sous lesquelles se manifestent les laves torrentielles sont finalement assez variables et souvent presque opposées : il peut y avoir beaucoup d'argile ou presque pas, elles peuvent faire beaucoup de bruit ou pas du tout, etc... Des points de convergence existent malgré tout évidemment: forte densité (de 1,5 à 2,4), fonctionnement transitoire, capacité à transporter de gros blocs, à générer des bourrelets latéraux ou frontaux, capacité érosive, etc... Cette diversité se lit aussi dans la difficulté à unifier la terminologie en langue anglaise, et à accorder les classifications.

En fait, si au lieu de travailler toutes laves confondues, on se cantonne à l'étude d'un bassin versant torrentiel et à ses laves torrentielles, l'unité dynamique du phénomène apparaît nettement comme on le voit bien dans l'excellente étude d'un torrent à laves célèbre, celui du Pousset (LEFEBVRE, 1979).

On est donc conduit à étudier le comportement dynamique des laves torrentielles, non pas toutes laves torrentielles confondues, mais en les différenciant selon leur composition. Les étudier signifie :

a) Déterminer les mécanismes physiques qui expliquent les particularités extérieures constatées (transport de gros blocs, fonctionnement en bouffées, écoulement en masse); or à l'heure actuelle plusieurs forces sont susceptibles d'expliquer ces particularités (turbulence, collision des particules, forces de liaison électrochimique, évasion du fluide interstitiel) et l'unanimité n'est pas encore faite sur la délimitation des champs d'intervention de ces différentes forces.

b) Déterminer les lois de comportement des fluides soumis à ces forces et élaborer les lois d'écoulement correspondantes.

c) Déterminer les critères qui permettront de savoir à quel type de lois de comportement on a affaire dans la réalité, pour un torrent déterminé et une lave déterminée.

Il est évident que ce travail est à faire ; il est néanmoins déjà entamé grâce à des recherches effectuées à l'étranger. 


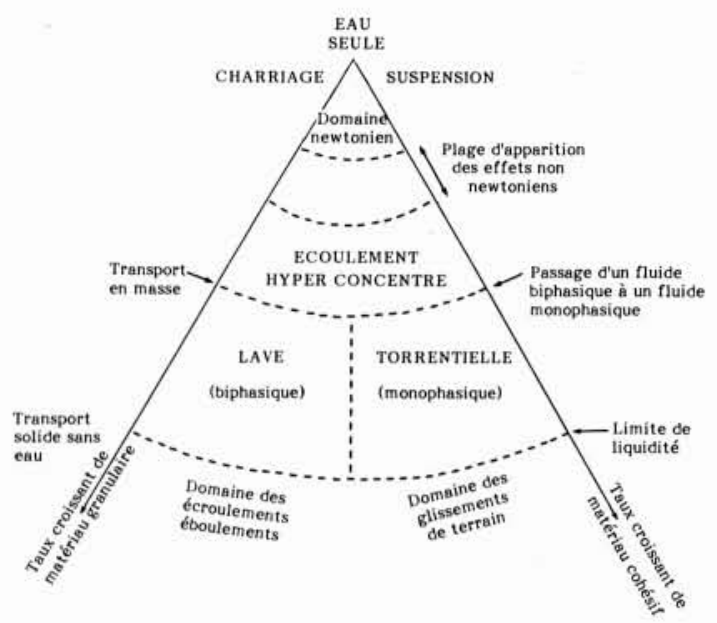

1. Représentation du champ d'étude de l'hydraulique torrentielle.

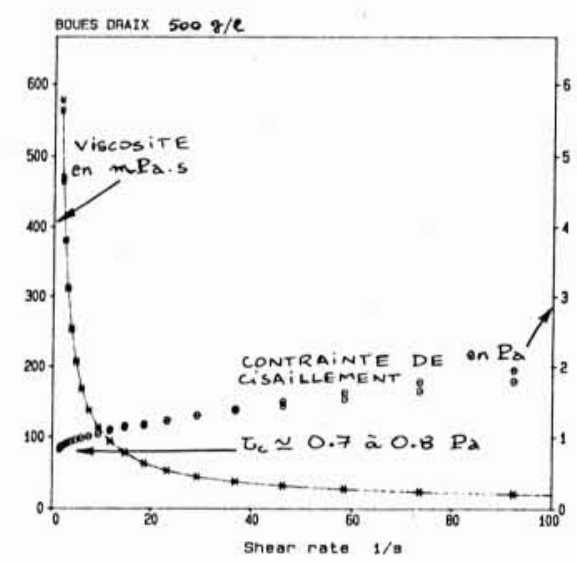

a)

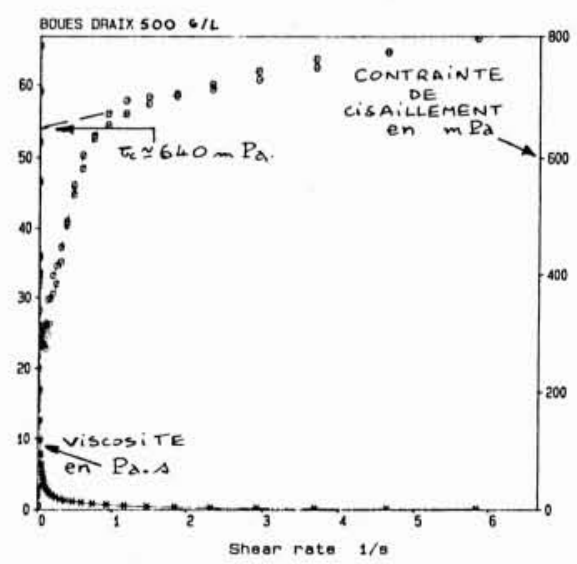

b)

2. Rhéogramme d'une boue à $500 \mathrm{~g} / \mathrm{l}$.

\subsection{Représentation du champ de l'hydraulique torrentielle}

Deux voies simplificatrices s'ouvrent manifestement au chercheur qui veut étudier l'effet d'une concentration importante en sédiments dans un écoulement: soit utiliser des matériaux fins, à tendance cohésive, qui donnent une suspension, puis une floculation et enfin un écoulement monophasique visqueux, soit utiliser des matériaux granulaires qui donnent un écoulement biphasique. On peut donc représenter (MEUNIER et al., $3^{\mathrm{c}}$ chapitre, 1987) le champ d'étude de l'hydraulique torrentielle dans un graphique triangulaire avec les trois pôles que sont l'eau, le matériau cohésif et le matériau granulaire (fig. 1).

Comme on le voit, ce graphique est entièrement qualitatif et permet seulement de situer les domaines d'étude des différents chercheurs. Il existe bien entendu une zone de transition sur chaque axe, où l'utilisation des lois de l'hydraulique usuelle cesse progressivement d'être licite ; il est plus simple de se placer d'emblée dans les zones où la concentration en matériau est telle qu'on est certain de ne plus être dans le domaine newtonien. C'est ce que nous allons faire en envisageant successivement chaque axe des matériaux pour les laves torrentielles.

\subsection{Lois d'écoulement des laves torrentielles en régime permanent}

\subsubsection{Axe des matériaux fins}

De nombreux chercheurs se sont penchés sur les modifications apportées aux caractéristiques d'une suspension lorsque sa concentration en matériau fin augmente, à commencer par EINSTEIN qui a calculé la variation de la viscosité de la suspension $\mu_{s}$ en fonction de la concentration volumique $C$ et a obtenu la formule bien connue :

$$
\frac{\mu_{s}}{\mu_{0}}=1+2,5 \mathrm{C} \text {. }
$$

Une synthèse de divers travaux peut être trouvée dans PFEIFF (1986), auquel on peut rajouter WAN ZHAOHUI (1982), et plus récemment O'BriEN et al. (1988) qui ont étudié la rhéologie d'échantillons de laves réelles. La plupart des résultats pour les matériaux cohésifs sont semblables à ceux de la figure 2(a) qui montre que la contrainte de cisaillement $(\tau)$ varie de façon à peu près linéaire en fonction du gradient de vitesse $\frac{\mathrm{d} u}{\mathrm{~d} y}$ au-delà d'un seuil $\tau c$. Le modèle de Bingham $\tau=\tau_{c}+\mu_{P} \frac{\mathrm{d} u}{\mathrm{~d} y}$ apparaît donc bien adapté pour représenter ce type de fluide. Comme de plus, il est assez simple d'expression, l'idée de l'utiliser pour modéliser les laves torrentielles apparaît séduisante. Il suffirait, pourrait-on penser, après avoir établi l'hydraulique à surface libre des fluides de Bingham, de déterminer avec un rhéomètre les deux paramètres $\left(\tau_{c}\right.$ et $\left.\mu_{P}\right)$ qui caractérisent le fluide, ou mieux, de connaître l'évolution de ces paramètres en fonction de la concentration en matériaux fins, pour qu'on puisse déterminer hauteur, vitesse, comportement de la lave, et connaître, par la même occasion, la 
quantité de sédiments qu'elle transporte. De nombreuses études existent qui donnent la variation de $\tau_{c}$ et $\mu_{P}$ en fonction de la concentration: DAI JILAN et al. (1980) cité dans O'BRIEN et al. (1988), WAN (1982), PFEIFF (1986), LCHF (1987). C'est sur l'élaboration d'une hydraulique des fluides de Bingham qui rendent compte des spécificités des laves torrentielles que les choses se compliquent.

JOHNSON (1970-1984) a été le pionnier dans l'exploration de cette voie ; du point de vue physique, il a estimé que la force de cohésion de la matrice argileuse était le facteur essentiel permettant d'expliquer les particularités de ces écoulements : portance de gros blocs, façonnage de vallée en $U$, etc... Il a élaboré les formules du régime permanent pour un plan semi-infini et pour un demicercle, cette forme de section étant destinée à mieux traduire l'effet des parois, particulièrement important avec un fluide visqueux; dans son document de 1970, il donne également la résolution du problème pour une section semi-elliptique, mais de façon erronnée, a-t-il l'honnêteté d'indiquer dans son document de 1984 ; le problème est en cours d'étude.

En conservant l'hypothèse d'un écoulement laminaire, on donne ci-dessous les équations du régime permanent, formulées à partir du paramètre $a$ :

$$
a=\frac{\tau_{c}}{\rho_{m} g h I}=\frac{\tau_{c}}{\tau_{0}}
$$

avec $\tau_{c}=$ rigidité initiale. $\rho_{m}=$ masse volumique du mélange. $I=$ pente.

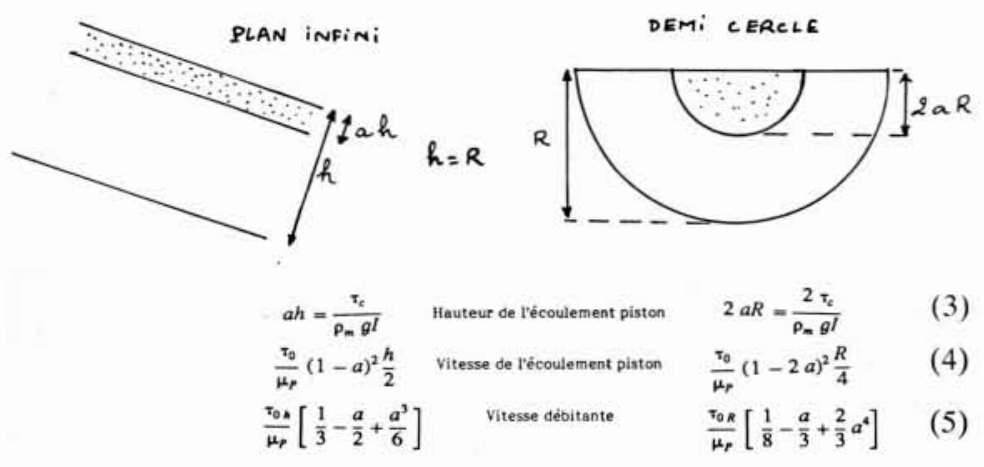

Comme on le voit, il y a une partie de l'écoulement où la contrainte de cisaillement ne peut vaincre la rigidité initiale : c'est l'écoulement piston (plug) qui est deux fois plus épais pour un demi-cercle que pour un plan infini et qui, surtout, est indépendant du débit ; si donc celui-ci décroît, c'est la partie inférieure de l'écoulement, avec cisaillement, qui rétrécit, diminue d'épaisseur et de vitesse, jusqu'à s'annuler pour une hauteur d'écoulement égale à celle de l'écoulement piston.

Ce modèle donne donc une explication rationnelle aux écoulements intermittents, par bouffées (fig. 3), que les Chinois rencontrent dans certains affluents du Fleuve Jaune (QUIAN et al., 1979, cité dans ENGELUND et WAN, 1984).

Ce phénomène a d'ailleurs été reproduit en laboratoire (ENGELUND et WAN ZHAOHUI, 1984), mais (significativement, nous y reviendrons) seulement avec une suspen-

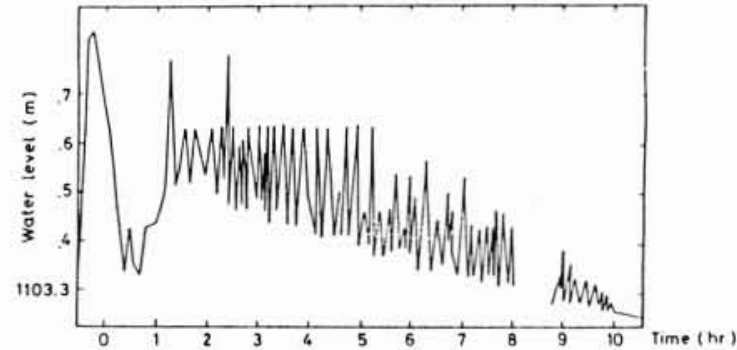

3. Variation du niveau d'eau du Fleuve Jaune lors 'd'un écoulement par bouffées.

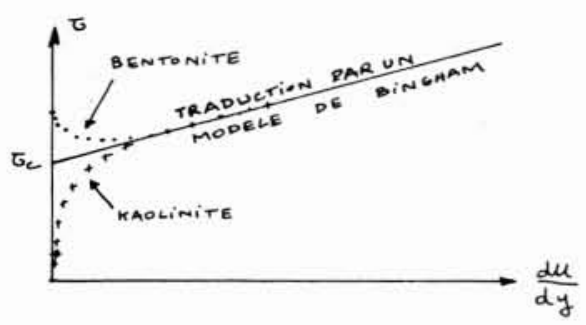

4. Forme des rhéogrammes expérimentaux de la bentonite et de la kaolinite.

sion de bentonite, et non avec de la kaolinite, dont les rhéogrammes diffèrent comme l'indique la figure 4 : si la bentonite a réellement une rigidité initiale, celle qu'on peut attribuer à la kaolinite n'est qu'un artefact et n'a pas une réelle signification physique ; il est logique qu'on ne puisse reproduire d'écoulements intermittents avec des fluides ayant un tel rhéogramme.

Quoi qu'il en soit, les formules indiquées ci-dessus permettent de retrouver les deux paramètres de la loi de Bingham $\left(\tau_{c}, \mu_{P}\right)$ à partir de mesures sur des écoulements réels, notamment dans deux circonstances :

- si on peut mesurer la vitesse et la largeur de l'écoulement piston, moyennant une hypothèse sur la forme de la section, on peut calculer $\tau_{c}$ et $\mu_{P}$;

- si on admet que les dépôts laissés par les laves torrentielles sont des écoulements piston qui se sont arrêtés, on peut retrouver $\tau_{c}$.

Il est dès lors intéressant de comparer les valeurs de ces paramètres à celles obtenues par des mesures directes sur des rhéomètres (RENDON VALENCIA, 1987). On constate alors (fig. 5) qu'elles diffèrent d'un ordre 2 à 3 pour $\tau_{c}$ et pour $\mu_{p}$.

Après cette constatation qui pose un sérieux problème quant à l'adéquation du modèle de Bingham aux laves torrentielles, résumons pour terminer les réserves qui lui sont faites ici et là et les questions qu'il faudrait élucider :

- L'hypothèse de base, que la matrice argileuse est responsable des caractéristiques des laves, est contestée pour les fluides naturels, souvent à granulométrie bimodale, pour lesquels à partir d'une certaine concen- 


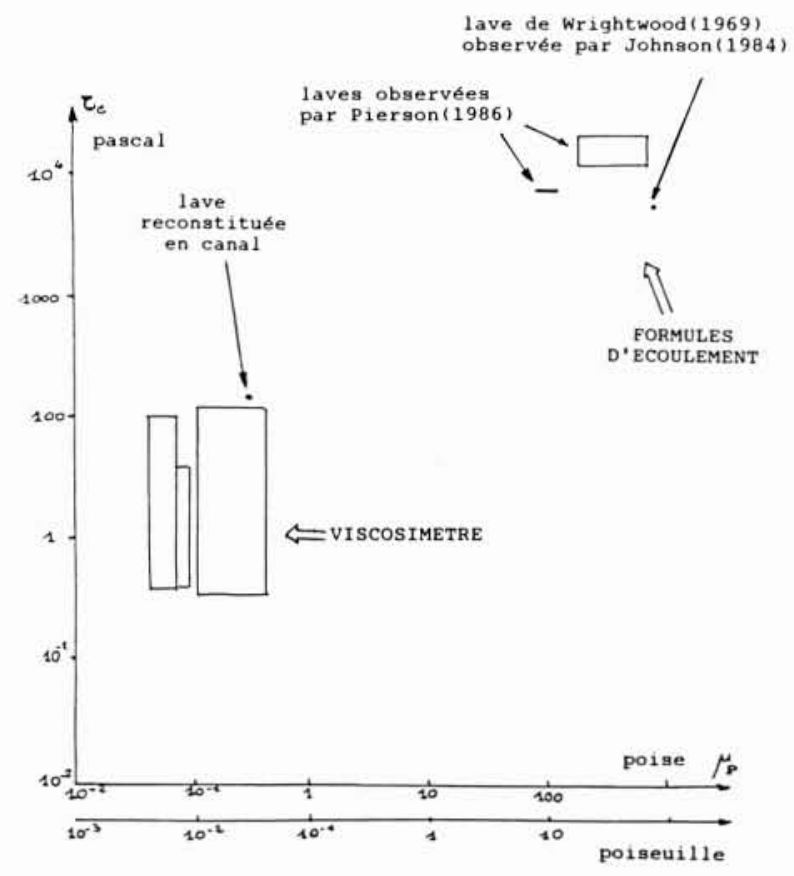

5. Valeurs des paramètres de la loi de Bingham obtenues au laboratoire ou à partir des formules d'écoulement.

tration, ce serait plutôt les collisions entre particules qui seraient le phénomène essentiel.

- Si l'on calcule le nombre de Reynolds spécifique aux fluides de Bingham (méthode de ToмiтA par exemple dans MidouX, 1985, p. 281), les écoulements seraient largement turbulents en adoptant des valeurs du modèle de Bingham tirées des mesures avec des rhéomètres, mais au voisinage de la transition entre laminaire et turbulent si on prend celles déduites de mesures sur des écoulements.

La question du type de comportement de ces écoulements est difficile car il faut faire des mesures internes à l'écoulement sans le modifier: l'existence d'une stratification, avec un écoulement piston en surface, et en dessous une couche turbulente encadrée par deux couches laminaires, est toutefois attestée pour des écoulements hyperconcentrés à faible pente (1\%) (WANG MiNG Fu et al., 1986).

- La réalité de l'existence d'un écoulement piston est contestée par les tenants de la solution « fluide granulaire "; la "preuve " expérimentale de son existence n'est en effet pas facile à faire.

- La traduction par un modèle de Bingham d'un rhéogramme réel est relativement approximative dans la gamme des gradients de vitesse qu'on rencontre en hydraulique torrentielle (inférieure à 10 ou $15 \mathrm{~s}^{-1}$ ) comme on peut le voir sur la figure 2(b) qui montre que la réalité physique d'une rigidité initiale est loin d'être prouvée.

- Avec cette modélisation, on ne s'intéresse qu'aux contraintes de cisaillement ; les laves torrentielles ont des surfaces d'écoulement bombées, ce qui indique des effets de contraintes normales non prises en compte avec le seul modèle de Bingham.

\subsubsection{Axe des matériaux granulaires}

Alors que jusqu'ici on a soigneusement évité les problèmes de terminologie, on est obligé, ici, d'ouvrir une parenthèse pour soulever une question : dans le contexte de l'ingénierie française, il semble admis de séparer la lave torrentielle, fluide monophasique très chargée en sédiments, qui aurait des effets catastrophiques, du charriage torrentiel, fluide biphasique, qui serait moins dangereux ; le côté monophasique de la lave est implicitement attribué à une matrice argileuse, rejoignant en cela le présupposé de JOHNSON (cf. 3.4.1) ; l'axe des matériaux granulaires, qui va nous occuper maintenant, s'intéresse aux fluides où la force responsable du comportement du fluide est la force "dispersive " de collision entre particules; les anglo-saxons appellent "grain flow " les écoulements de solides secs et "debris flows" les écoulements naturels eau + sédiments, où les sédiments grossiers jouent le rôle moteur au point de vue comportement du fluide. Comme on a constaté que des laves torrentielles peuvent avoir extrêmement peu d'argile (SAURET, 1986) que beaucoup de recherches récentes (DAviES, 1988) rendent secondaire le rôle de l'argile par rapport à celui des matériaux grossiers dès qu'ils sont en quantité suffisante, j'utiliserai ici le terme "lave torrentielle" pour traduire celui de "debris flows "; le critère " monophasique " n'est pas forcément supprimé ; il doit simplement être compris dans un sens plus large: lorsqu'il y a énormément de sédiments solides qui s'écoulent " en masse ", la latitude de chacun d'eux de bouger par rapport à son voisin est faible et l'ensemble de l'écoulement a une apparence monophasique.

On peut maintenant en venir aux travaux effectués sur cet axe des matériaux granulaires, en commençant par le pionnier, BAGNOLD $(1954,1956)$ qui a étudié le comportement rhéologique de suspensions de billes fortement concentrées; il a d'abord fait une étude théorique en séparant a priori deux domaines; celui où la quantité de mouvement reçue par un grain après un choc est absorbée par la viscosité du fluide (régime macro-visqueux) et celui où elle est essentiellement transférée à un autre élément solide (régime inertiel). Dans le premier, la loi de comportement est newtonnienne, dans le deuxième c'est celle des fluides dilatants $\left(e n(\mathrm{~d} u / \mathrm{d} y)^{2}\right)$ qu'il faut utiliser. Il a vérifié la validité de ses deux analyses par expérimentation avec un rhéomètre et l'a trouvée bonne pour des concentrations allant de $13 \%$ à $60 \%$. Les deux domaines sont en fait séparés par une zone de transition délimitée par les valeurs des nombres adimensionnels dits de Bagnold.

L'analyse de Bagnold est intéressante en ce sens que la concentration des sédiments intervient directement dans l'expression de la loi de comportement à travers la "concentration linéaire " $\lambda$ qui est le rapport entre le diamètre moyen des grains solides et la distance moyenne qui les sépare (fig. 6).

En faisant ces travaux, BAGNOLD ne visait pas particulièrement le domaine des laves torrentielles; il a toute- 


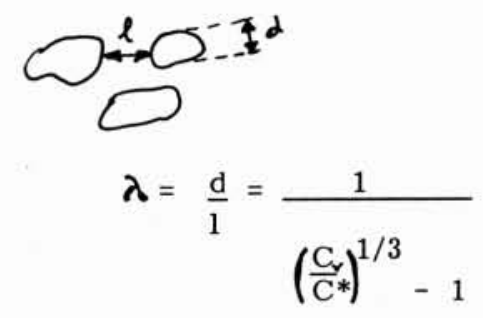

\section{Concentration volumique \\ C* Compacité}

6. Définition de la concentration linéaire.

fois utilisé ses résultats pour expliciter une coulée de gravier apparemment sec (article de 1954) et les boues de particules fines (article de 1956) en les situant dans les deux cas dans le domaine macro-visqueux. Dans notre domaine, c'est TAKAHASHI (1978-1981) qui va se servir de ces résultats pour élaborer la théorie la plus complète à ce jour, relative aux laves torrentielles (debris flow) puisqu'il a étudié le déclenchement, la propagation, l'étalement, l'arrêt et la vitesse d'érosion des laves torrentielles; dans ce paragraphe consacré au régime permanent, on se contente d'indiquer la formule que l'on obtient par intégration de la loi de comportement de Bagnold en régime inertiel avec $\tau$ contrainte de cisaillement et $\sigma$ contrainte normale.

$$
\begin{aligned}
\tau & =a_{i} \sin \alpha \cdot \rho_{s}\left(\lambda \cdot d_{50}\right)^{2}\left(\frac{\mathrm{d} u}{\mathrm{~d} y}\right)^{2} \\
\sigma & =\tau / \operatorname{tg} \alpha
\end{aligned}
$$

où $\alpha$ est l'angle de frottement interne dynamique, $\rho_{s}$ la masse volumique du sédiment, et $d_{50}$ le diamètre médian en poids. $a_{i}$ est un paramètre de calage qui est égal à 0,042 avec les expériences de Bagnold. On obtient :

$$
V=k\left(\frac{\rho_{m}}{\rho_{s}}\right)^{1 / 2}\left(\frac{g}{a_{i} \sin \alpha}\right)^{1 / 2} \frac{1}{\lambda \cdot d_{50}} I^{1 / 2} h^{3 / 2}
$$

où $k$ est un paramètre numérique d'intégration qui vaut $2 / 5$ pour un plan semi-infini et $2 / 7$ pour un demi-cercle.

En pratique, cette formule s'avère difficile d'emploi avec deux types de difficulté :

- les paramètres intrinsèques à la loi de Bagnold sont mal connus : $\alpha$ est l'angle de frottement interne dynamique ; BAGNOLD l'a trouvé de l'ordre de $18^{\circ}$ par comparaison entre contrainte normale et tangentielle dans le régime inertiel et à $37^{\circ}$ dans le régime macro-visqueux ; mais surtout $a_{i}$ n'a pas encore une valeur stabilisée : égal à 0,042 dans le test rhéologique de Bagnold, il a été pris égal à 0,5 par TAKAHASHI (1980) au début de ses expérimentations sur modèle réduit alors qu'il est plutôt de l'ordre de 0,065 maintenant (TAKAHASHI et al., 1987) ;
- mais surtout les paramètres de terrain $\rho_{m}, \lambda$ et $d_{50}$ sont très difficiles à appréhender finement, et comme ils ne sont pas indépendants entre eux, il n'est pas possible de les fixer "au juger». En conséquence la formule (8) a un caractère prédictif médiocre, de même d'ailleurs que la formule (5) qui en est l'équivalent pour fluide de Bingham.

\subsubsection{Autres approches}

D'autres approches ont été tentées, notamment celle de DELEON et JEPPSON (1982) qui restent dans le cadre des fluides newtoniens, admettent que les écoulements sont laminaires et se donnent deux relations reliant les variables de comportement interne du fluide (densité $\rho_{m}$ et viscosité $\mu$ ) et les variables d'écoulement (rayon hydraulique $R$ et coefficient de perte de charge de Chézy K).

$$
\begin{aligned}
K & =1,02 \operatorname{Re}^{0.52} \\
\rho_{m} / \mu & =10 / R .
\end{aligned}
$$

Ces deux relations ont été contrôlées sur les rares données existantes et devraient être encore vérifiées avant d'être considérées comme très valables, d'autant que l'estimation des viscosités qui permettent de caler la formule dépend de l'hypothèse faite sur le comportement du fluide.

Quoi qu'il en soit, ce modèle, que DELEON et JEPPSON utilisent surtout pour compléter un modèle de ST VENANT conduit à une formule très simple de régime permanent :

$$
V=11,4 \cdot R \cdot I .
$$

On trouve par ailleurs dans la littérature d'autres formules qui sont proposées pour calculer la vitesse des laves torrentielles. Par exemple, en France, THIERY (1914) a proposé de réduire le coefficient de rugosité des formules classiques grâce à un facteur torrentiel $k$

$$
k=\frac{1}{1+C_{v}\left(\frac{\rho_{s}}{\rho}-1\right)} .
$$

En URSS, Shribny (cité dans TAKahashi, 1981) a proposé :

$$
V=6,5 R^{2 / 3} I^{1 / 4} \frac{1}{\left[\frac{\rho_{s}}{\rho} \cdot \frac{\left(\rho_{s}-\rho\right) C}{\left(\rho_{s}+\rho\right)(1-C)}+1\right]^{1 / 2}} .
$$

Des approches purement empiriques existent aussi : ainsi PIERSON (1986) sur dix mesures, propose pour le front de lave :

$$
V=1,5+6 R . I .
$$

\subsection{Etudes des laves torrentielles en transitoire}

En fait, si par la force des réflexes acquis, on a souvent tendance à commencer l'étude d'un phénomène par celle de son régime permanent, les laves torrentielles sont d'abord des phénomènes transitoires, et il est possible de 
les prendre d'emblée comme tels. DAVIES (1988) s'est justement interrogé sur la raison de ce comportement par bouffées et il propose une classification en trois domaines :

Le premier, où les écoulements sont très chargés en sédiments mais où on ne voit pas de comportement par bouffées.

Le deuxième, où le fluide contient en lui-même l'explication de son comportement par bouffées; ce phénomène se rencontre principalement dans les régions loessiques de Chine dont on a déjà parlé ; mais DAVIES n'accepte pas l'explication donnée ci-dessus par l'existence d'un écoulement piston dû à une loi de comportement avec rigidité initiale ; il propose une explication du type matériau granulaire, mais dans le domaine macrovisqueux de Bagnold, la viscosité de la matrice argileuse servant, selon lui, justement à faire basculer le fluide du comportement inertiel vers le comportement macro-visqueux. Une étude expérimentale très intéressante le conduit à assimiler les bouffées des laves torrentielles dans ce domaine aux rouleaux (roll-waves) qui se produisent en écoulements d'eau claire lorsque le nombre de Froude dépasse 2. L'explication physique serait la suivante : pour de tels écoulements, fluide et sédiments se déplacent à la même vitesse, en masse; or dans le domaine macro-visqueux, quand la contrainte de cisaillement augmente, la capacité de transport de sédiments diminue (pour une raison mal expliquée), ceci ne peut se traduire par un dépôt des sédiments les plus gros comme dans le cas du charriage classique, puisque tout se déplace en masse, mais est réalisé par une diminution de la vitesse globale; ce ralentissement permet au fluide amont de venir accroitre la hauteur d'eau, donc d'augmenter la contrainte de cisaillement, donc de diminuer la vitesse, etc...

Le troisième domaine est celui des laves torrentielles à une seule bouffée (il peut se produire plusieurs laves torrentielles à une seule bouffée, bien sûr) où la cause du comportement transitoire est extérieure au fluide : soit le déclenchement lui-même de la bouffée (glissement de berges ou de versant), soit une embâcle temporaire suivie d'une rupture ; c'est le domaine expérimenté par TAKAHASHI qui lui permet, au point de vue modélisation mathématique, d'utiliser les équations de ST VENANT avec comme loi de perte de charge soit celle de Manning Strickler, soit celle de Bagnold dans le domaine inertiel (Eq. (8)) comme il l'a fait pour la lave torrentielle du Nevado del Ruiz qui a détruit la ville d'Armero le 13 novembre 1985 (TAKAHASHI et al., 1987). Le problème devient donc celui de trouver la loi de perte de charge adaptée au type d'écoulement: par exemple, TAKAHASHI fixe à 30 la limite du rapport $h / d_{50}$ en dessous de laquelle il faut prendre la loi dilatante (Eq. (8)).

On peut prolonger cette optique et utiliser les outils classiques de modélisation numérique des écoulements transitoires pour reconstituer le parcours des laves torrentielles, à la condition d'avoir assez d'observations de terrain pour caler un tant soit peu le modèle: par exemple, la lave torrentielle du 24 juillet 1988 sur le Verdarel de St Chaffrey a laissé des traces visibles qui nous ont permis de caler globalement un modèle de propagation: deux possibilités ont été testées (VILAMEUNIER, travail en cours) avec une perte de charge usuelle du type Manning Strickler, et avec une perte de charge supplémentaire du type loi de Coulomb. Dans le premier cas, le coefficient de rugosité est égal à 6 et semble donc irréaliste; dans le deuxième, on peut s'imposer un coefficient de rugosité égal à 20 et l'angle de frottement interne est égal à $12^{\circ}$. Dans les deux cas, il suffit d'une lachure instantanée d'un volume relativement faible $\left(5000\right.$ à $\left.10000 \mathrm{~m}^{3}\right)$ de coulée boueuse pour retrouver un front de lave de $3,5 \mathrm{~m}$ de haut s'écoulant à une vitesse de 3 à $5 \mathrm{~m} / \mathrm{s}$. L'intérêt de la deuxième méthode d'introduction des pertes de charge est qu'elle permet de simuler l'arrêt d'une coulée.

Comme on s'en doute, le gros problème est l'obtention de données de terrain permettant de caler le modèle ; comme ces données sont rarement des données fines (vitesses instantanées, hauteurs au cours du temps), le calage ne peut être que global, comparable en cela à la méthode utilisée par SOGREAH pour caler un modèle physique de laves torrentielles (SOGREAH, 1985). Il faudra de toute façon le caler sur un grand nombre de phénomènes réels, plus ou moins bien observés, avant qu'on puisse passer à l'étape suivante qui consisterait à s'en servir pour prédéterminer des phénomènes, le côté aléatoire du déclenchement semblant en effet pour l'instant irréductible, puisqu'il est de nature hydrologique.

\subsection{L'hydraulique torrentielle en dehors des laves torrentielles}

Si les laves torrentielles sont les phénomènes les plus spectaculaires et les plus dommageables qui se produisent dans les torrents, l'ingénieur de terrain est le plus clair de son temps confronté à des phénomènes de moins grande ampleur, qui nous rapprochent de l'hydraulique des crues classiques, sauf que le transport solide, quand il y en $\mathrm{a}$, peut devenir très important ( 1 à $20-40 \%$ en volume). Une meilleure connaissance de cette hydraulique là (le premier domaine de DAVIES, cf. 2.5) est donc très important, avec au moins trois questions concrètes immédiates: le seuil de dépavage, le régime uniforme et le transport solide.

\subsubsection{Contrainte critique de cisaillement et pavage}

La notion de pavage d'un torrent est bien connue dans le milieu des torrents ; elle permet d'expliquer l'existence de crues d'eau claire; mais elle est perçue comme une sécurité à court terme qui rend le long terme problématique : si une crue est assez forte pour dépaver le torrent, est-ce que cela ne va pas engendrer une catastrophe? Des moyens de quantifier le dépavage seraient donc hautement appréciés; ceux-ci semblent ne pas réellement exister à l'heure actuelle ; les travaux faits dans une optique de détermination de seuils, l'ont été dans l'optique classique de trouver le seuil de cisaillement critique. Citons, entre autres (BATHURST et al., 1985) une formule simple (le débit critique de début de transport est en $\left.\mathrm{m}^{3} / \mathrm{s} . \mathrm{m}\right)$ :

$$
\frac{q_{c}}{g^{1 / 2} \cdot d_{50}^{3 / 2}}=0,15 \cdot I^{-1.12} \text {. }
$$



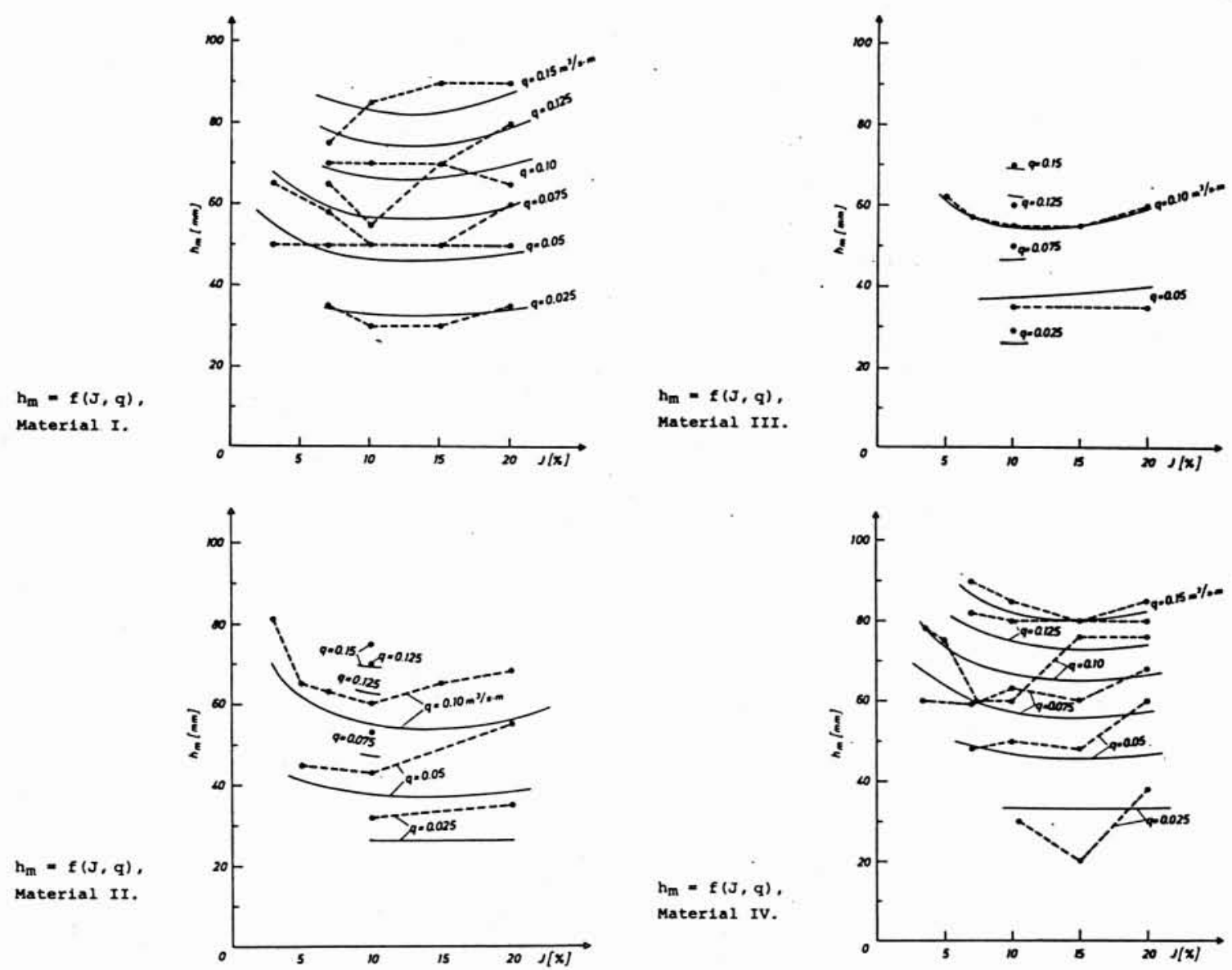

7. Croissance de la hauteur d'eau en fonction de la pente (dans Smart et Jaeggi, 1983).
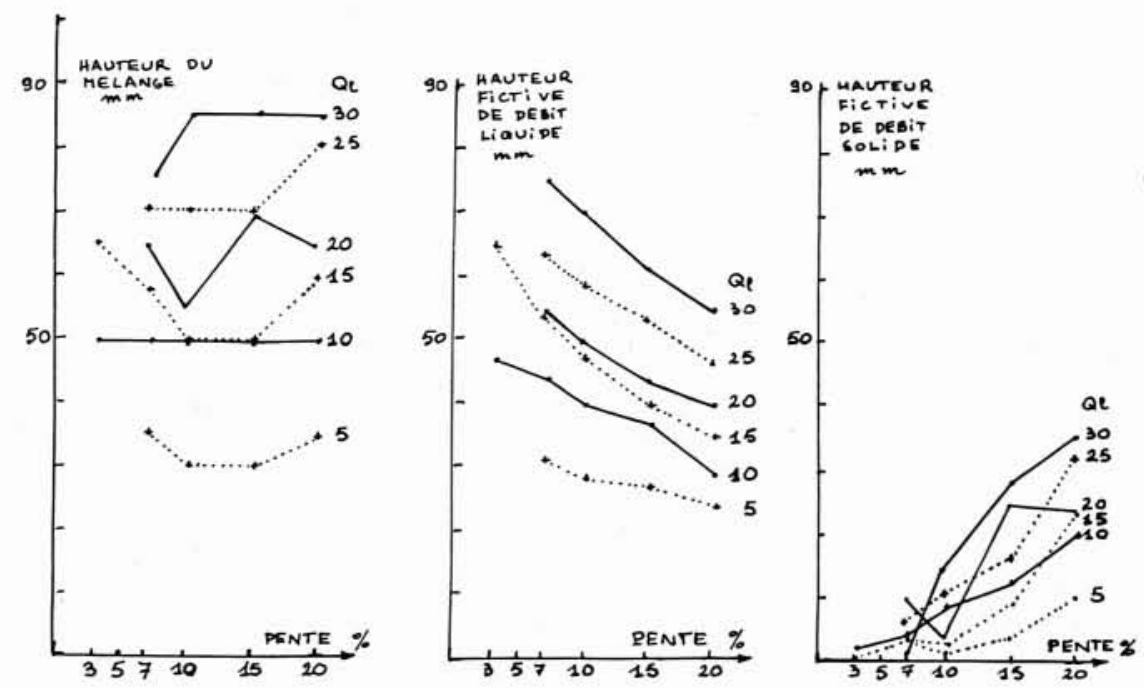

8. Hauteurs fictives de débit solide, de débit liquide et hauteur de mélange en écoulements torrentiels, à débit liquide constant (données de Smart et Jaeggi, 1983. Matériau 1). 
Cette formule a été établie sur modèle réduit pour des pentes inférieures à $9 \%$ et sur du matériau remanié mono-disperse ; l'utiliser au cas d'un torrent pavé n'est donc pas évident ; il faut d'abord admettre qu'elle est valable, ensuite déterminer sur quelle partie du lit du torrent pavé on va calculer la courbe granulométrique, et enfin posséder une méthode de terrain commode permettant de déterminer cette courbe granulométrique.

$\mathrm{Si}$ cette formule (15) est indiquée ici, c'est parce qu'elle est très simple d'emploi; il en existe d'autres exprimées sous la forme classique du critère de Shields, indiquant une éventuelle croissance de la contrainte critique en fonction de la pente (MizuYama, 1977, ASHIDA et al., 1977 cité dans TAKAHASHI, 1987). La comparaison faite par TAKAHASHI (1987) montre que l'accord avec la formule (15) est acceptable.

\subsubsection{Concentration, hauteur d'eau et perte de charge}

Si l'on regarde les travaux effectués en matière de régime uniforme et de transport solide, on constate qu'il y a de nombreuses études qui traitent séparément soit un problème, soit lautre. Or des expériences (SMART et JAEGGi, 1985) montrent qu'à débit liquide constant la hauteur d'eau décroît puis croît en fonction de la pente ; cette augmentation est due à l'importance du transport solide qui ne peut plus être négligé dans les formules de régime uniforme (fig. 7).

Aux formules ne tenant pas compte de cette conséquence, on préférera donc la méthode de SMART et JAEGGI (1983), malgré sa complexité : $C$ est le rapport $q_{s} / q_{\ell}, R F$ est le rayon hydraulique relatif au fond selon la méthode d'EINSTEIN de prise en compte des effets de paroi, $\theta$ et $\theta_{C R}$ sont les contraintes effective et critique en valeurs réduites, $Z_{90}$ est la hauteur relative $\left(Z_{90}=\right.$ $\left.R F / d_{90}\right)$

$$
\begin{gathered}
V_{\ell=2,5} \sqrt{g \cdot R F \cdot I} \cdot\left(1-e^{-\frac{0,05 \cdot Z_{90}}{\sqrt{l}}}\right)^{1 / 2} \times \\
\times \log _{\ell}\left(8,2 \cdot Z_{90}\right) \\
C=\frac{4}{\left(\frac{\rho_{s}}{\rho}-1\right)}\left(\frac{d_{90}}{d_{30}}\right)^{0.2} I^{1.6}\left(1-\frac{\theta_{C R}}{\theta}\right) \\
q_{\ell=} V_{\ell} \cdot R F\left\{1-1,41\left[\frac{C \cdot q_{\ell}}{\left(\frac{\rho_{s}}{\rho}-1\right) \cdot g \cdot d_{50}^{3}}\right]^{0.18} \cdot I^{1.14}\right\} .
\end{gathered}
$$

Ces formules ont été calées à partir de résultats obtenus en canal, en régime permanent et avec recyclage du matériau. Elles donnent l'état d'un écoulement torrentiel à la capacité maximale de transports, puisque en deçà se produit une érosion du fond du lit, et au-delà, se produit un dépôt. D'autres essais et d'autres formules sont bien entendu proposés par d'autres chercheurs : on peut en trouver quelques-unes dans TAKAHASHI (1987).

Relation entre vitesse du fluide et pente : Si l'on rassemble des formules donnant le régime uniforme dans le domaine des torrents, on constate que pour aucune d'entre elles, la pente ne joue le même rôle qu'en hydraulique classique ; c'est-à-dire à la puissance 0,5 comme dans les formules de Chézy ou de Manning Strickler : ( $R$ est le rayon hydraulique, $h_{m}$ la hauteur du mélange, et $D$ la profondeur (Depth) : $D=S / L$ où $S$ est la section et $L$ la largeur au miroir).

- Formule de JARRETT (1984) :

$$
V=3,81 \cdot I^{0.12} \cdot R^{0,83} \quad N=75 .
$$

- Régressions de l'auteur :

Données in situ de BATHurst (1985):

$$
\begin{aligned}
& V=1,3 \cdot I^{-0,084} \cdot D^{0.863} R^{2}=0,79 N=44 \\
& V=\frac{3,9}{\left(d_{84}\right)^{0.56}} I^{0.289} \cdot D \quad R^{2}=0,87 \quad N=44 .
\end{aligned}
$$

- Données en canal de SMART et JAEGGi (1983) :

$$
V=7\left(\frac{h_{m}}{d_{90}}\right)^{0.35} \cdot I^{0.3} \cdot h_{m}^{0.5} R^{2}=0,8 \quad N=71 .
$$

Explicitation des composantes liquides et solides dans la hauteur de l'écoulement: On peut tenter de comprendre pourquoi le rôle de la pente dans ces équations est modifié par rapport à l'hydraulique fluviale; la figure 7 indique comme raison plausible la présence de transport solide, d'où l'idée de séparer les composantes solides et liquides de la hauteur de l'écoulement : par exemple, en reprenant les données de SMART et JAEGGI (fig. 7) et en calculant une hauteur fictive de l'écoulement liquide $h_{\ell}$ (égale au rapport entre le débit liquide connu et la vitesse du liquide calculée par la méthode du sel), on obtient un résultat conforme à la théorie hydraulique classique (cf. fig. 8), c'est-à-dire une décroissance de la hauteur avec la pente à débit liquide constant.

Une régression multiple sur les données de SMART et JAEGGI dans ce nouveau cadre donne:

$$
\frac{u_{\ell}}{\sqrt{g d_{50}}}=16,2 \cdot I^{0.54} \cdot\left(\frac{h_{\ell}}{d_{50}}\right)^{0,73} R^{2}=0,88 \mathrm{~N}=71 .
$$

Ceci nous permet d'adopter la formule classique de Chézy, mais pour l'écoulement liquide seulement :

$$
u_{\ell}=K \sqrt{h_{\ell} \cdot I} \text { avec } K=14\left(\frac{h_{\ell}}{d_{50}}\right)^{0.25} .
$$

Il faut maintenant compléter par une estimation de la hauteur fictive de l'écoulement solide; à partir des mêmes données et avec une imprécision de $\pm 40 \%$, on peut proposer :

$$
\begin{aligned}
\frac{h_{s}}{h_{\ell}} & =9,65 \cdot I^{1,7} \\
\frac{h_{s}}{d_{50}} & =\left(\frac{q_{s}}{\sqrt{g \cdot d_{50}^{3}}}\right)^{1 / 2} .
\end{aligned}
$$

La hauteur totale de l'écoulement est alors obtenue par la somme des deux hauteurs; la formule (25) est plus difficile d'utilisation puisqu'elle oblige à connaitre $q_{s}$; mais elle est peut-être prometteuse si on peut vérifier 


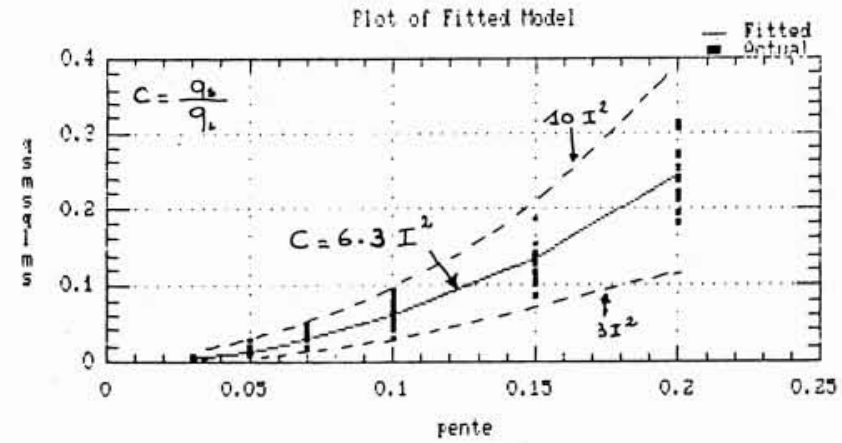

9. Régression curviligne sur les données de transport solide de Smart et Jaeggi.

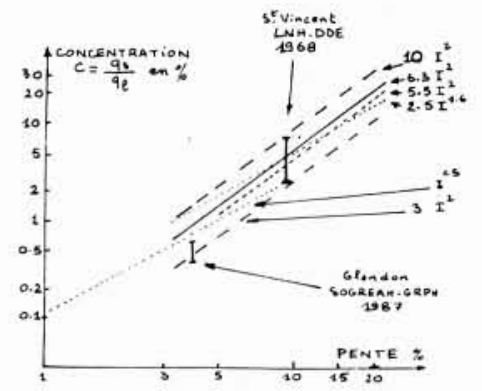

10. Formules simplifiées donnant le transport solide.

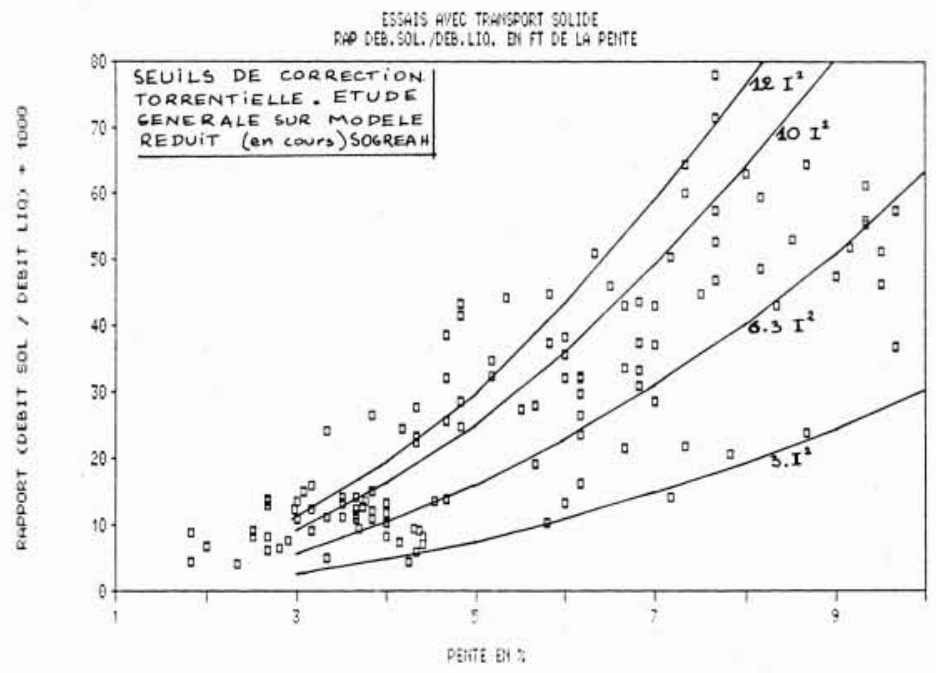

11. Variation de la concentration en fonction de la pente.
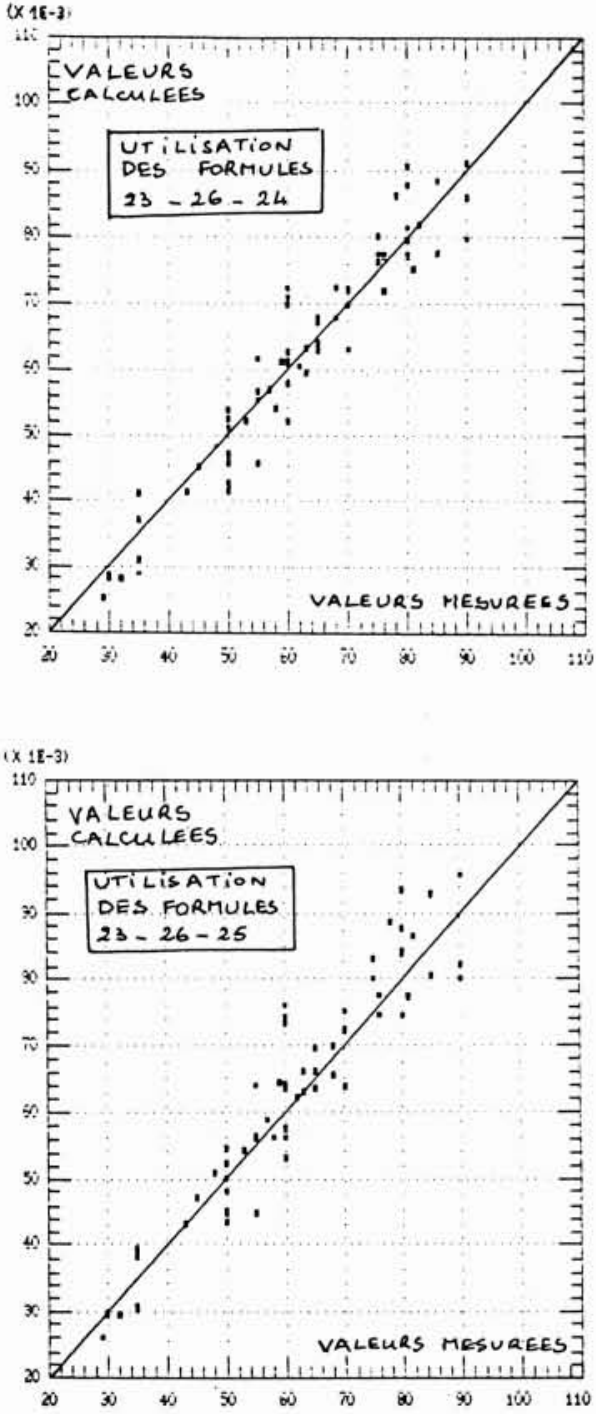

12. Comparaison entre les valeurs calculées et mesurées de hauteur de mélange. 
qu'elle reste valable en dehors de la capacité maximale de transport, alors que la formule (24) ne le sera certainement plus.

\subsubsection{Ordre de grandeur de la concentration $\left(q_{s} / q_{\ell}\right)$}

Conformément aux formules d'hydraulique fluviale donnant le transport solide, celles qui sont proposées dans le domaine des torrents font intervenir les caractéristiques granulométriques et le seuil de début du transport; les premières sont mal connues et le deuxième est souvent négligeable par rapport à la contrainte de cisaillement des épisodes torrentiels catastrophiques. On peut donc se placer à un niveau plus sommaire de précision donnant seulement un ordre de grandeur : le paramètre explicatif le plus important est la pente ; les formules trouvées dans la littérature sont les suivantes:

$\begin{array}{lll}\text { SMART et JAEGGi (1983) } & C=2,5 & I^{1,6} \\ \text { MizUYAMA (1981) } & C=5,5 & I^{2} \\ \text { SHOKLITSCH-BATHURST et al. (1985) } & C=\frac{2,5}{\rho_{s} / \rho} & I^{1,5} .\end{array}$

De plus, la régression curviligne sur les données de SMART et JAEGGI (1983) donne (fig. 9) :

$$
C=6,3 I^{2.02} \quad R^{2}=0,921 \quad N=71
$$

encadrées par $C=10 I^{2}$ et $C=3 I^{2}$ (fig. 9). Ces formules "encadrantes " englobent aussi les formules simplifiées des autres auteurs indiquées ci-dessus (fig. 10), des résultats expérimentaux ponctuels ( $\mathrm{LNH}$ et SOGREAH, fig. 10), et des résultats expérimentaux plus systématiques (SOGREAH, étude en cours, fig. 11) présentés ici toutes hauteurs confondues.

On peut donc utiliser la formule (26) à $\pm 50 \%$ près, dans les cas où on cherche un ordre de grandeur du transport solide à la capacité maximale de transport, et qu'on peut négliger la contrainte critique de cisaillement mais, vraisemblablement, seulement au-delà d'une pente de $3 \%$ à $5 \%$. Lorsque la contrainte critique et la rugosité relative ne sont pas négligeables, il faut revenir aux formules complètes mais se pose alors le problème du choix de celle qui convient le moins mal. Soulignons d'ailleurs à nouveau que toutes ces données sont obtenues sur modèle réduit avec recyclage du sédiment ce qui pose le problème bien connu du tri granulométrique.

\subsubsection{Conclusion}

Les paragraphes précédents nous ont permis de dégager, à partir des travaux de SMART et JAEGGI, un ensemble de formules simplifiées qui permettent de calculer, lorsqu'on connait le débit liquide, un ordre de grandeur des variables utiles dans les projets de correction torrentielle :

- la hauteur fictive du débit liquide par la formule de Chézy $U_{\ell}=K \sqrt{h_{\ell} \cdot I}$ avec :

$$
K=14\left(\frac{h_{\ell}}{d_{50}}\right)^{0.25}
$$

- le débit solide par la formule simplifiée (26):

$$
q_{s} / q_{\ell}=6,3 \cdot I^{2}
$$

ou par une formule un peu plus complexe :

$$
q_{s} / q_{\ell}=4,6 I^{2,1}\left(\frac{h_{\ell}}{d_{50}}\right)^{0.23} R^{2}=0,95
$$

- la hauteur fictive du débit solide par les formules ou (25)

$$
\frac{h_{s}}{h_{\ell}}=9,65 \cdot I^{1.7} \text { ou } \frac{h_{s}}{d_{50}}=\left(\frac{q_{s}}{\sqrt{g d_{50}^{3}}}\right)^{0.5} .
$$

Les graphiques de la figure 12 montrent l'écart qui existe entre les valeurs mesurées et les valeurs calculées en utilisant la formule (26) pour le transport solide et tour à tour la formule (24) et la formule (25) pour la hauteur fictive de débit solide.

En y ajoutant la formule de Bathurst qui donne le débit critique de début de transport (formule (15)), le projeteur peut approcher de manière quantitative ce qui se passe dans un torrent ; insistons toutefois sur le fait que ces formules devront être confirmées par de nouvelles expériences et par la pratique, vu les nombreuses incertitudes dont nous avons parlé.

\section{Conclusion}

Les deux disciplines scientifiques présentées dans cette synthèse n'en sont pas au même degré de développement : pour permettre la quantification, l'érosion torrentielle dépend des mesures disponibles et celles-ci ne sont pour l'instant disponibles que sur des bassins versants expérimentaux situés sur des Terres Noires des Alpes du Sud; un ensemble de relations reliant pluie, débit liquide et débit solide commence à y être élaboré qui permettra à terme, la prédétermination de l'érosion des espaces dégradés de même nature géologique; par contre, dans les Alpes du Nord ou les terrains de montagne à géologie différente, faute de mesures, seules les approches naturalistes ou empiriques peuvent être utilisées, rendant difficile le dimensionnement des projets.

L'hydraulique torrentielle est moins tributaire du "terrain" que l'érosion torrentielle, et les travaux de recherches conduits à l'étranger peuvent donc être utilisés ailleurs; de plus, cette discipline autorise les travaux sur modèles réduits; en conséquence, il est enfin possible de commencer la clarification du champ de I'hydraulique torrentielle et d'y délimiter les domaines où tel phénomène plutôt que tel autre joue le rôle principal; il est également possible de proposer des formules qui peuvent aider les ingénieurs de terrain à dimensionner leurs projets même si leur marge d'incertitude est importante.

La route est encore longue évidemment avant que ces disciplines soient réellement devenues des ensembles de méthodes couramment utilisées en ingénierie, mais un tournant est peut-être en train de se franchir qui les fait passer du rang de sciences qualitatives à celui de disciplines quantificatrices. 


\section{Bibliographie}

Ahida, Takahashi, Mizuyama (1977), Study of the initiation of motion of sand mixtures in a steep slope channel. SHIN-SABO. Vol. $29, \mathrm{~N}^{\circ} 4,1977$.

BAGNOLD (1954), Experiments on a gravity free dispersion of large solid spheres in a Newtonian fluid under shear. Philosophical transactions of the Royal Society of London. Series A. Vol. 225.

BAGNOLD (1956), The flow of cohesionless grains in fluids. Philosophical transactions of the Royal Society of London. Serics A. Vol. 249.

Bathurst, Graf, huu Hal CaO (1985), Bed load discharge equations for steep mountain rivers. International Workshop on Problems of Sediment Transport in Gravel Bed Rivers. Pingree Park Colorado, USA.

BATHURST (1985), Flow resistance estimation in mountain rivers. Journal of Hydraulic Engineering. Vol. $111, \mathrm{~N}^{\circ} 4$, avril 1985.

BERNARD (1927), Cours de restauration des montagnes. Ecole Nationale des Eaux et Forêts, Nancy.

Buffalo, Oliveros et Quelennec (1988), L'érosion des terres noires dans la région du Buëch (Hautes-Alpes). Contribution à l'étude des processus érosifs sur le bassin versant représentatif de Saint-Genis. BRGM, Marseille. La Houille Blanche, Nㅜ 3/4-1989.

C.E.M.A.G.R.E.F. (1984), Méthodologie de programmation des actions de restauration des terrains en montagne. Le cas de la Maurienne. Groupement de Grenoble. Février 1984.

Colas, Dumolard (1988), Erodabilité des marnes. CETE, Aix-en-Provence.

DAI JILAN et al. (1980), An experimental study of slurry transport in pipes. Proc. Int. Symposium on River Sedimentation.

DAVIES (1988), Debris flow surges. A laboratory investigation. Mitteilungen der Versuchsanstalt für Wasserbau; Hydrologie und Glaziologie. Zurich.

DELEON et JEPPSON (1982), Hydraulics ans numerical solutions of steady state but spatially varied debris flow. Utah Water Research Laboratory, Hydraulics and Hydrology Series. UWRL/H-82/03.

Egels, Kasser, Muxart, Meunier, Guet (1988), Utilisation des méthodes topographiques pour la mesure de l'érosion des marnes et comparaison aux mesures de transport solide. IGN, CNRS, CEMAGREF. La Houille Blanche, $\mathrm{N}^{\circ} 3 / 4-1989$.

ENGELUND et WAN ZHAOHUI (1984), Instability of hyperconcentrated flow. Journal of Hydraulics Engineering. ASCE, Vol. 110, $\mathrm{N}^{\circ} 3$, mars 1984.

Guigo (1978), Le Val Parma: Essai de cartographie des potentialités d'érosion sur un bassin de l'Apennin septentrional. Méditerranée. $\mathrm{N}^{\circ} 3,1978$.

JARRET (1984), Hydraulics of High, gradient streams. Journal of Hydraulic Engineering. Vol. 110, $\mathrm{N}^{\circ} 11$, novembre 1984.
JoHnson (1970), Physical processes in geology. Freeman Cooper and Company. San Francisco, USA.

JOHNSON with contributions by RODINE (1984), Debris flow. In "Slope Instability ", edited by Brunsden and Prior. John Wiley and Sons.

LCHF (1987), Synthèse des connaissances sur le tassement et la rhéologie des vases. LCHF, Service Technique Central de la Direction des Ports et des Voies Navigables.

Laboratoire National d'Hydraulique (EDF) (1968), Correction du St-Vincent.

LEFEBVRE (1979), Etude hydraulique de la protection de la RN 6 au confluent de l'Arc et du Torrent du Pousset. SOGREAH, DDE, Savoic.

MATHYS, MEUNier (1988), Mesure et interprétation du processus d'érosion dans les Marnes des Alpes-du-Sud, à l'échelle de la petite ravine. CEMAGREF, Grenoble. La Houille Blanche, N $N^{*}$ 3/4-1989.

Meunier, Mathys (1986), Etude de bassin, le St-Antoine. CEMAGREF, mars 1986.

meunier, Cambon, Mathys, Olivier, Coulmeau (1987), Premier compte rendu de recherches des bassins versants expérimentaux de Draix. CEMAGREF, ONF, RTM.

MEUNIER (1988), Bassins versants expérimentaux de Draix. Effet des travaux RTM sur les crues torrentielles. Exemple du bassin du Brusquet. Hydrologie Forestière. CEMAGREF, ONF, RTM.

Mıdoux (1985), Mécanique et rhéologie des fluides en génie chimique. Librairie Lavoisier.

Mizuyama (1977), Bed load transport in steep channels. Kyoto University, Japan.

Mizuyama (1981), An Intermediate phenomenon between debris flow and bed load transport. AIHS, Publ. n* 132.

mura, Cambon, Combes, Meunier, Olivier (1988), La gestion du Bassin Versant Expérimental de Draix pour la mesure de l'érosion. Symposium on sediment budgets. AIHS, décembre 1988. Porto Alègre, Brésil.

O'BRIEN et JULIEN (1988), Laboratory analysis of mudflow propertics. Journal of hydraulics engineering. Vol. 114, $\mathrm{N}^{*} 8$, août 1988.

OKUNISHI et SUWA (1985), Hydrological approach to debris flow. International Symposium on Erosion, debris flow and disaster prevention. Tsukuba, 1985.

PFEIFF (1986), Dépendance de la trainée d'obstacles cylindriques, de la rhéologie de suspensions aqueuses concentrées. Thèse USMG, INPG.

Pierson (1986), Flow Behavior of channelized debris flows. Mount St-Helen. Washington in * Hillslopes Processes », 1986, Boston, Allen et Unwin, Abrahams Ed.

Quian Ning, WaN et Quian (1979), The flow with heavy sediment concentration in the Yellow River. Journal of Quing hua University. Vol. 19, N*2, 1979.

RENDON VALENCIA (1987), Les modèles rhéologiques et la résistance hydraulique en torrents. DEA, IMFS, CEMAGREF. 
SAURET (1986), Synthèse bibliographique sur les laves torrentielles. CETE, Aix-en-Provence.

SMART et JAEGGI (1983), Sediment transport on steep slopes. Mitteilungen der Versuchsanstalt für Wasserbau, Hydrologie und Glaziologie, Zurich.

SOGREAH (1985), Etude hydraulique du franchissement du torrent de la Guiaz par la voie express Le Fayet, Les Houches. DDE Savoic, janvier 1985.

SOGREAH, GRPH Alpes (1987), Etude hydraulique du Glandon. Essais sur modèle réduit du cône de déjections.

TAKAHASHI (1978), Mechanical characteristics of debris flow. ASCE, Journal of the Hydraulics Division. Vol. 104, $\mathrm{N}^{*} \mathrm{Hy} 8$, août 1978 .

TAKAHASHI (1980), Debris flow on prismatic open channel. ASCE, Journal of Hydraulics Division. Vol. 106, $\mathrm{N}^{\circ}$ Hy 3, mars 1980.

Takahashi (1981), Debris flow. Ann. Rev. Fluid. Mech. 1981, 13.
TAKAHASHI (1987), High Velocity flow in steep erodible channels. AIRH, $22^{e}$ Congrès, Lausanne.

Takahashi, Nakagawa, Kuang (1987), Estimation of debris flow hydrograph on varied slope bed. Corvallis Symposium, AIHS.

THIERY (1914), Restauration des montagnes. Librairie Polytechnique Ch. Béranger, éditeur.

VILA, MEUNIER (travail en cours), Essai d'application de la modélisation numérique des laves torrentielles par les équations de St-Venant au cas du Verdarel de St-Chaffrey. CEMAGREF.

WAN ZHAOHUI (1982), Bed materiels movement in hyperconcentrated flow. Series Paper $n^{\circ} 31$. Institute of hydrodynamics and hydraulic engineering. Technical University of Denmark.

Wang Ming Fu, Duan Wenzhong, Tan Guang Ming et ZHAN YIZHENG (1986), On the structure and movement mechanism of flow with hyperconcentration of sediment. International Worshop on Flow at Hyperconcentration of Sediment. Septembre 1986, Beijing, China.

Adresse de l'auteur :

Monsieur Maurice Meunier

CEMAGREF, BP 76

38402 St-Martin-d'Hères

Tél. (16) 76540072 


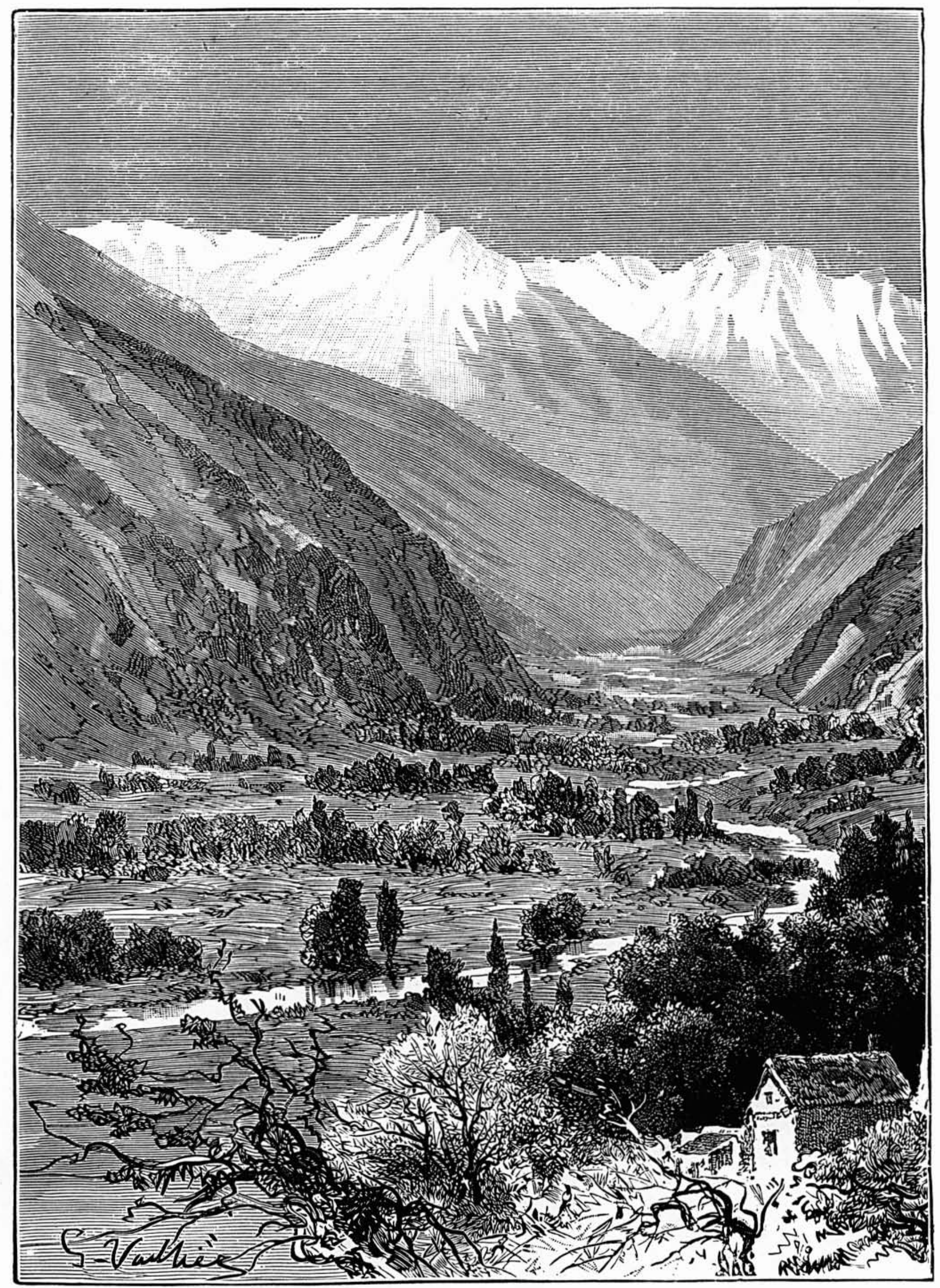

LA HOUILLE BLANCHE/ $\mathrm{N}^{\circ}$ 5-1989 